\title{
ASaiM-MT: a validated and optimized ASaiM workflow for metatranscriptomics analysis within Galaxy framework
}

\section{[version 1; peer review: 1 approved with reservations]}

\author{
Subina Mehta (D1), Marie Crane (D1), Emma Leith1, Bérénice Batut(iD2, \\ Saskia Hiltemann ${ }^{3}$, Magnus $\varnothing$ Arntzen4, Benoit J. Kunath (iD), Francesco Delogu 4 , \\ Ray Sajulga1, Praveen Kumar¹, James E. Johnson1, Timothy J. Griffin (iD1, \\ Pratik D. Jagtap (iD) \\ 1 University of Minnesota, Twin Cities, MN, 55455, USA \\ 2Department of Bioinformatics, University of Freiburg, Georges-Köhler-Allee 106, Freiburg, Germany \\ ${ }^{3}$ Department of Pathology, Erasmus Medical Center, Rotterdam, The Netherlands \\ ${ }^{4}$ Norwegian University of Life Sciences, Ås, 1430, Norway
}

V1 First published: 11 Feb 2021, 10:103
https://doi.org/10.12688/f1000research.28608.1

Latest published: 19 Apr 2021, 10:103

https://doi.org/10.12688/f1000research.28608.2

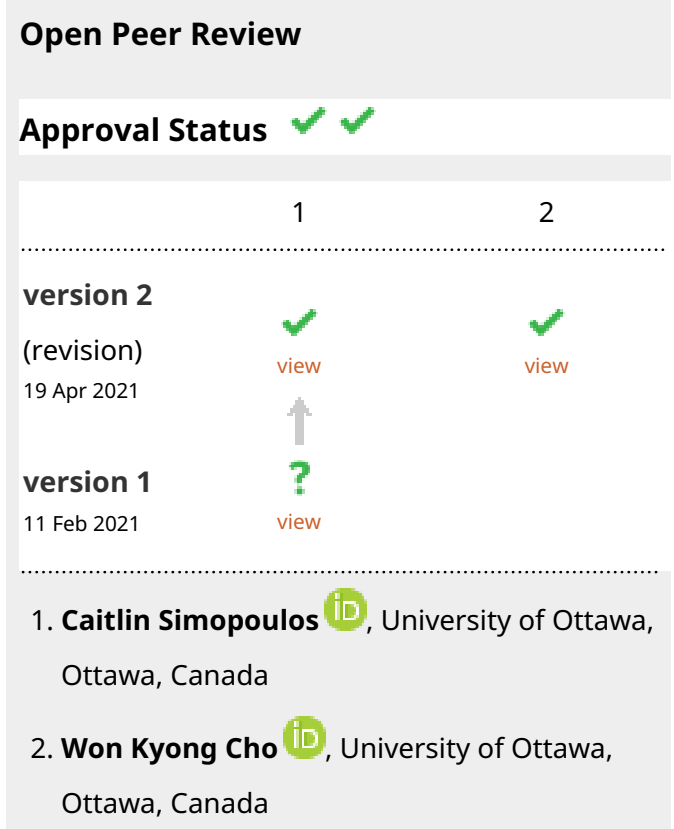

Any reports and responses or comments on the article can be found at the end of the article. 
that will enable researchers to understand dynamic functional response of the microbiome in a wide variety of metatranscriptomics studies. This improved and optimized ASaiM-metatranscriptomics (ASaiM-MT) workflow is publicly available via the ASaiM framework, documented and supported with training material so that users can interrogate and characterize metatranscriptomic data, as part of larger meta-omic studies of microbiomes.

Keywords

Galaxy, metatranscriptomics, microbiome, functional analysis

This article is included in the Galaxy gateway.

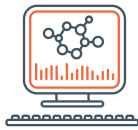

This article is included in the Bioinformatics

gateway.

Corresponding authors: Subina Mehta (smehta@umn.edu), Pratik D. Jagtap (pjagtap@umn.edu)

Author roles: Mehta S: Formal Analysis, Investigation, Software, Validation, Writing - Original Draft Preparation, Writing - Review \& Editing; Crane M: Software, Validation; Leith E: Writing - Review \& Editing; Batut B: Investigation, Software, Validation, Writing - Review \& Editing; Hiltemann S: Methodology, Software, Validation, Writing - Review \& Editing; Arntzen Mø: Data Curation, Formal Analysis, Funding Acquisition, Writing - Review \& Editing; Kunath BJ: Data Curation, Writing - Review \& Editing; Delogu F: Data Curation, Writing Review \& Editing; Sajulga R: Methodology, Software; Kumar P: Methodology, Software; Johnson JE: Methodology, Software; Griffin TJ Conceptualization, Funding Acquisition, Supervision, Writing - Review \& Editing; Jagtap PD: Conceptualization, Project Administration, Supervision, Writing - Original Draft Preparation, Writing - Review \& Editing

Competing interests: No competing interests were disclosed.

Grant information: We acknowledge funding for this work from the grant National Cancer Institute - Informatics Technology for Cancer Research (NCI-ITCR) grant 1U24CA199347, National Science Foundation (U.S.) grant 1458524 to T.J.G and a grant through the Norwegian Centennial Chair (NOCC) program at the University of Minnesota to T.J.G and M.A. The European Galaxy server that was used for data analysis is in part funded by Collaborative Research Centre 992 Medical Epigenetics (DFG grant SFB 992/1 2012) and German Federal Ministry of Education and Research (BMBF grants 031 A538A/A538C RBC, 031 L0101B/031L0101C de.NBI-epi, 031L0106 de.STAIR (de.NBI)).

The funders had no role in study design, data collection and analysis, decision to publish, or preparation of the manuscript.

Copyright: $\odot 2021$ Mehta S et al. This is an open access article distributed under the terms of the Creative Commons Attribution License, which permits unrestricted use, distribution, and reproduction in any medium, provided the original work is properly cited.

How to cite this article: Mehta S, Crane M, Leith E et al. ASaiM-MT: a validated and optimized ASaiM workflow for metatranscriptomics analysis within Galaxy framework [version 1; peer review: 1 approved with reservations] F1000Research 2021, 10:103 https://doi.org/10.12688/f1000research.28608.1

First published: 11 Feb 2021, 10:103 https://doi.org/10.12688/f1000research.28608.1 


\section{Introduction}

Understanding the role of microbiome in patho-physiological conditions such as inflammatory diseases, obesity, and cancer has opened up various avenues of research ${ }^{1}$. Experimental design and biological interpretation of microbiome data has become an area of intense focus as the contributions to human health and disease are becoming clearer $^{2,3}$. The 'meta-omics' approaches, such as metagenomics, metatranscriptomics and metaproteomics have been developed to study microbiomes without culturing and target the major macromolecules that constitute the community, namely DNA, RNA and proteins. While metagenomics (16S rRNA or whole genome sequencing) focuses on the taxonomy profile and functional potential ${ }^{4}$, metatranscriptomics, metaproteomics and meta-metabolomics ${ }^{5}$ uncover the functional response of the microbiome to stimuli on the short and long time-scale, respectively ${ }^{6,7}$.

Metatranscriptomics has been used to analyze microbial gene expression profiles from a variety of complex sample types, e.g. human microbiome, aquatic or terrestrial environments, plant-microbe interactions ${ }^{8}$. Despite these applications, challenges still exist in the analysis of the complex metatranscriptomics data. Metatranscriptomics data is usually generated using highthroughput sequencing of short RNA-Seq reads using Illumina sequencing technology (PMID: 30298254). Many software tools and workflows are available for metatranscriptomics analysis. These include tools for RNA-Seq Data Preprocessing: Quality Control (FastQC), Ribosomal RNA removal (SortMeRNA, barrnap), host RNA removal (BMTagger), De Novo Assembly (Trinity, MetaVelvet, Oases, IDBA-MT, TAG), Transcript
Taxonomy (Kraken, GOTTCHA, MetaPhlAn2), Functional Annotation (HUMAnN2), Annotation of assembled contigs are subjected to gene finding programs such as FragGeneScan followed by functional assignment using DIAMOND searches against KEGG, NCBI RefSeq, UniProt. Differential Expression analysis is performed by tools such as EdgeR, DeSeq2 and limma. "Reads-Based" analysis is performed by tools such as MetaTrans, COMAN, FMAP, SAMSA2, ASaiM and Assembly Based analysis: SqueezeMeta, IMP, MOSCA. Some of these open source tools ${ }^{9}$ have been incorporated within the Galaxy bioinformatics workbench ${ }^{10}$ to make it more accessible to users on a single platform.

ASaiM framework was previously developed by Batut et al. to perform metagenomics and metatranscriptomics data analysis $^{11}$. The major goal of ASaiM was to develop an accessible, resharable, and user-friendly framework for microbiome researchers, implemented within the Galaxy platform ${ }^{12}$. The framework integrates a comprehensive set of microbiota related tools, predefined and tested workflows as well as supporting training material and documentation. It is available for users as a Docker image but also as a web server (https://metagenomics.usegalaxy.eu/). This implementation also enables flexibility, so that the workflow can be customized for datasets of diverse origin as new software tools or methods emerge. To address the need for optimizing ASaiM for metatranscriptomics data, we added the ASaiM-metatranscriptomics (ASaiM-MT) (Figure 1), a metatranscriptomics workflow, and rigorously tested it to ensure reliable analysis of metatranscriptomics data. Our testing and validation focused on using contemporary tools in their

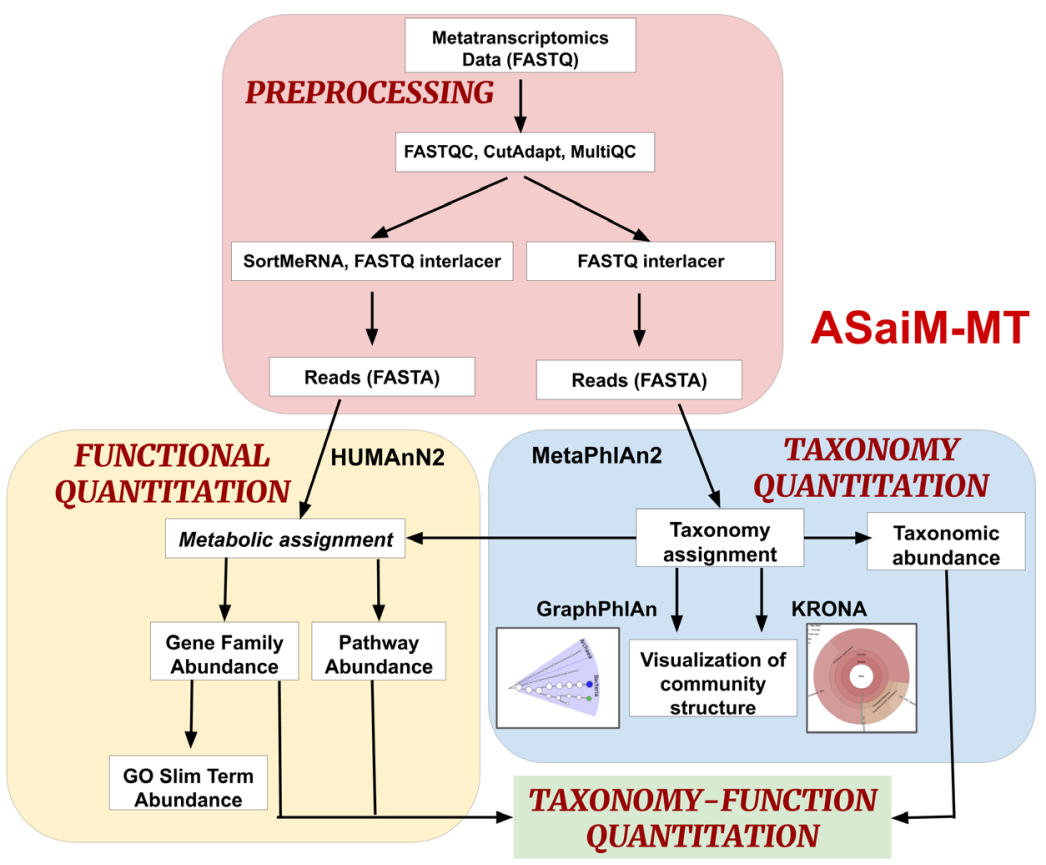

Figure 1. ASaiM-MT workflow: The workflow is divided into 4 parts. (i) Preprocessing: Process raw metatranscriptomics data to perform further analysis. (ii) Taxonomy Quantitation: Assignment of taxonomy along with abundance values and visualization. (iii) Functional Quantitation: metabolic assignment of identified functions and gene and pathway abundance annotation. (iv) Taxonomy-Function Quantitation: combine taxonomy and functional quantitation values into relative abundance values at different levels such as e.g., the abundance of a pathway between phyla. 
most current version (Table 1), capable of handling large datasets, and ensuring that the outputs from each of the tools were compatible in order to build an integrated and automated workflow. The workflow also has potential for integration with other meta-omic tools and workflows in Galaxy, such as those designed for metaproteomics ${ }^{13}$, to enable multi-omic data analysis.

The ASaiM-MT workflow is available via the ASaiM framework, specially at https://metagenomics.usegalaxy.eu/), for users to test their metatranscriptomics data. It is supported by a step-by-step tutorial (link), available on the Galaxy Training Network (GTN) (citation), which provides explanation for the different steps and the opportunity for online, hands-on training in using the workflow, with a trimmed dataset.

\section{Methods}

Input

Our optimized ASaiM-MT workflow (available via https:// metagenomics.usegalaxy.eu/) accepts Illumina paired end FASTQ sequence files (Forward read and Reverse read). As an alternative, a single-end FASTQ sequence can also be used as an input, with minor modifications in the downstream processing tool (such as changing the sequence type in CutAdapt and Filter with SortmeRNA as single end reads and also removing the FASTQ interlacer tool).

\section{Workflow implementation}

The ASaiM-MT workflow contains all the processing steps and the parameters required for the metatranscriptomics analysis from RNA-Seq data collected under a single biological condition. This workflow is also compatible with the single-end sequencing reads although parameters have to be changed to accommodate this input. This workflow is a multi-step analysis with preprocessing/ data cleaning, taxonomy analysis and functional analysis. The starting data input for the workflow are the FASTQ files - forward and reverse reads (obtained from the Illumina sequencer). We describe below the tools and their functions in the workflow. For comparative analysis of multiple biological conditions, the users have an option to use the MT2MQ tool to generate inputs for statistical analysis (see discussion).

Table 1. Enhancements in the ASaiM-MT workflow as compared to the original ASaiM shotgun metagenomics workflow.

\begin{tabular}{|c|c|c|c|}
\hline Tool Function & ASaiM Shotgun Metagenomics & ASaiM-MT & Updates \\
\hline \multirow{2}{*}{ Quality control } & \multirow{2}{*}{ FASTQC } & FASTQC & Version change $(0.69 \rightarrow 0.72)$ \\
\hline & & MultiQC & Tool added \\
\hline Adapter Trimming & TrimGalore! & CutAdapt & Tool replaced \\
\hline Dereplication & vsearch & - & \\
\hline rRNA selection & FilterwithSortmeRNA & FilterwithSortmeRNA & Version change $(2.1 b .4 \rightarrow 2.1 b .6)$ \\
\hline Interlacing & FASTQ-Join & FASTQ interlacer & Tool replaced \\
\hline Taxonomic assignation & MetaPhIAn2 & MetaPhIAn2 & No change \\
\hline $\begin{array}{l}\text { Formatting for the } \\
\text { different taxonomic } \\
\text { levels }\end{array}$ & Format MetaPhIAn2 & Format MetaPhIAn2 & No change \\
\hline Functional assignation & HUMAnN2 & HUMAnN2 & No change \\
\hline \multirow{4}{*}{ Visualization } & Export to GraPhIAn & Export to GraPhIAn & Parameters changed \\
\hline & Krona pie chart & Krona pie chart & Version change $(2.6 .1 \rightarrow 2.6 .1 .1)$ \\
\hline & GraPhIAn & GraPhIAn & No change \\
\hline & $\begin{array}{l}\text { Generation, personalization and } \\
\text { annotation of tree }\end{array}$ & $\begin{array}{l}\text { Generation, personalization and } \\
\text { annotation of tree }\end{array}$ & No change \\
\hline Regroup to GO terms & Group abundances & Group abundances & Tool updated \\
\hline $\begin{array}{l}\text { Unpack Pathway } \\
\text { abundance }\end{array}$ & - & $\begin{array}{c}\text { Unpack Pathway abundance to } \\
\text { show gene families }\end{array}$ & Tool added \\
\hline $\begin{array}{l}\text { Extracting Gene level } \\
\text { information }\end{array}$ & - & Create gene level families file & Tool added \\
\hline Text manipulation tools & - & Select, Sort, Group & Tools added \\
\hline
\end{tabular}




\section{1) Preprocessing}

Occasionally, sequencing can introduce incorrect identification of nucleotides and these errors can lead to misinterpretation of the data, thus bringing in the need to preprocess (Figure 2(a)) the data before analysis. The first step in our analysis is to perform quality control to remove such sequencing errors. For this, we use FastQC to assess the quality of each sample and MultiQC to combine each result into a single report. To improve the quality of the data, CutAdapt was used to trim low-certainty bases from reads, filter out reads of poor quality or short length, unwanted sequences, including adapters, primers, and poly-A tails. The ASaiM-metagenomics shotgun workflow uses Trim Galore! for trimming of adapters. Trim Galore! works as a wrapper that includes CutAdapt and FastQC. However, for the ASaiM-MT workflow we chose CutAdapt ${ }^{14}$ for adapter trimming because it is more error tolerant, processes fast and modifies and filters reads according to user's preference compared to TrimGalore!. Next, SortMeRNA ${ }^{15}$ was used to remove any rRNA sequences, which are often used for easy taxonomic characterization of microbiomes but do not provide functional information. We eliminated the step of de-replication of reads (V-Search) in the ASaiM-MT workflow, in order to retain the multiple copies of sequences for metatranscriptomics quantitation. The final step in cleaning and processing the data is to interlace the forward and reverse reads since the following steps require a single file per sample. For performing this action, the original ASaiM Shotgun workflow used the FASTQ-joiner to join the reads. However, in the ASaiM-MT version, we use the FASTQ interlacer. FASTQ interlacer joins the forward (/1) and the reverse reads (/2) using the sequence identifiers; sequences without designation will be named as single reads. The reason ASaiM-MT uses FASTQ-interlacer rather than FASTQ-joiner is because the joiner tool combines the forward and reverse read sequence together while the interlacer puts the forward and reverse read sequences in the same file while retaining the entity of each read along with an additional file with unpaired sequences. We perform the interlacing on the data both before and after the SortMeRNA step since the following steps require both data with and data without rRNA.

\section{2) Extraction of taxonomic profile}

To understand a microbial community, we must first understand which organisms are present along with their abundance. There are several approaches to microbial taxonomic profiling,
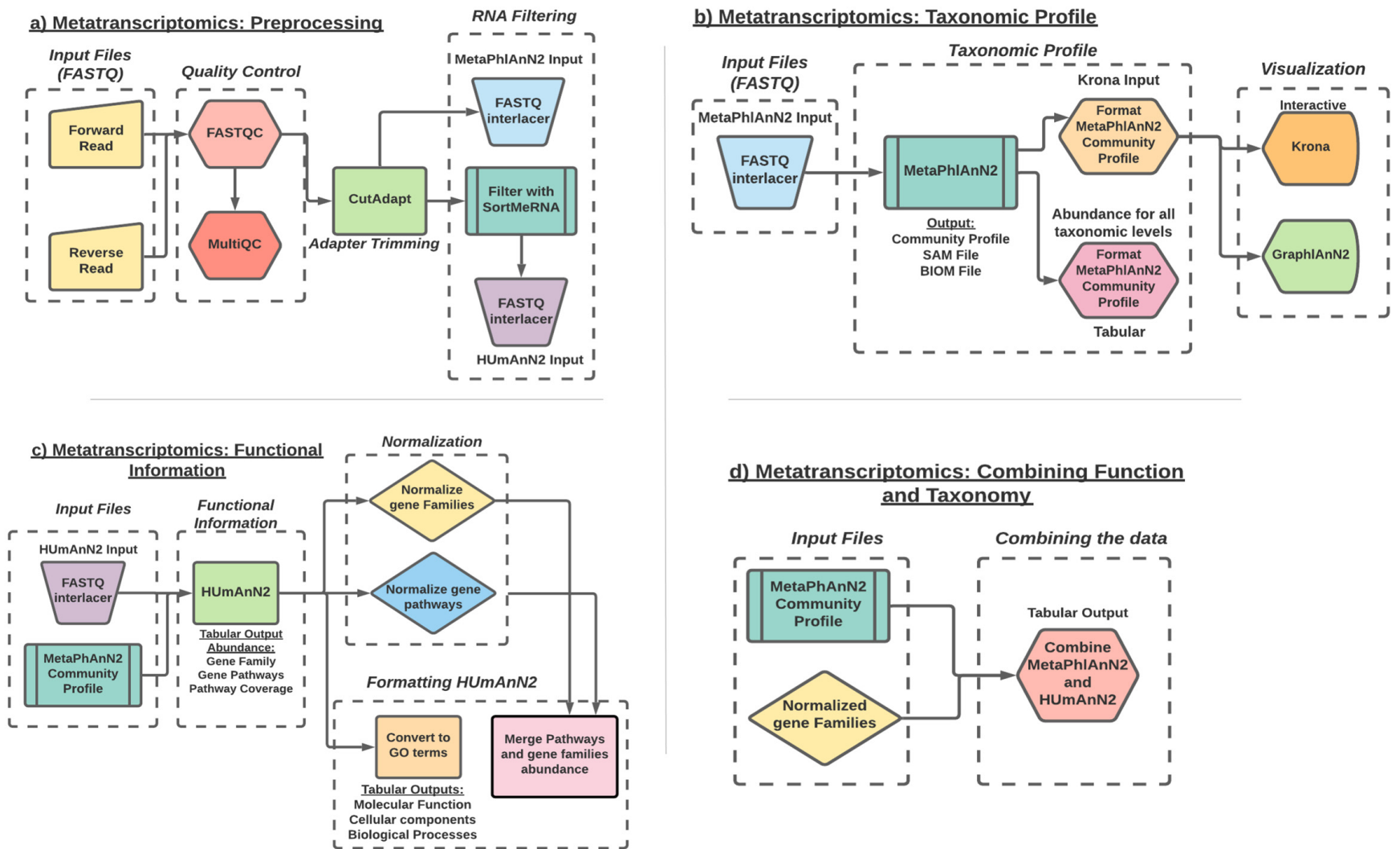

\section{d) Metatranscriptomics: Combining Function} and Taxonomy

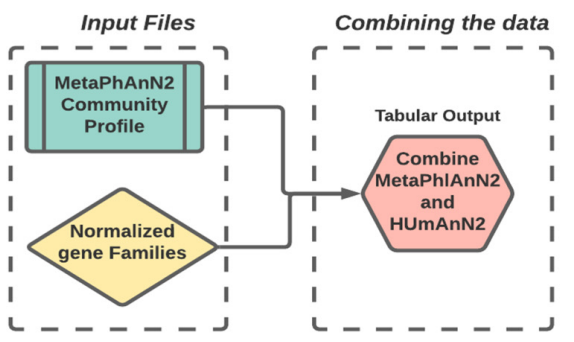

Figure 2. Diagram of the ASaiM-MT workflow: a) Preprocessing workflow: Workflow representation of the tools involved in quality check, data trimming and RNA filtering. b) Taxonomic profile workflow: workflow representation of taxonomy assignment tool (MetaPhlAn2) and post processing of the data using the Format MetaPhIAn2 tool. The workflow includes visualization of the data using interactive Krona and GraPhIAn plots. c) Functional information workflow: representation of tools involved in functional annotation (HUMAnN2), normalization of the data d) Combine Functional-Taxonomy abundance workflow: workflow representing tools that combines (Combine MetaPhIAn2 and HUMAnN2 outputs) and groups (Group abundances into GO slim terms) the functional and taxonomy output. 
but this workflow (Figure 2(b)) uses the marker gene approach. MetaPhlAn2 checks every read against a database of approximately one million clade-specific marker genes from nearly 17,000 reference genomes (bacterial, archaeal, viral, and eukaryotic $)^{16}$. For this particular step, we use all reads, including rRNA since they are useful for taxonomic profiling. The outputs of MetaPhlAn2 are a SAM (sequence alignment map) file and a BIOM (Biological Observation Matrix) file, which both show the mapping of reads onto the reference database, and Community Profile tabular output which contains information regarding the taxa present and their relative abundance. This table includes information at all taxonomic levels, so to parse it out into each separate level we use the Format MetaPhlAn2 tool.

We use two different tools to visualize the taxonomic profiles. First, we use $\mathrm{Krona}^{17}$, which creates an interactive pie chart from the hierarchical taxonomic data. This chart is multilayered for each taxonomic level and can be zoomed for viewing at each level. GraPhlAn ${ }^{18}$ is the other visualization tool, which creates a publication-ready circular representation of a phylogenetic tree based on the taxonomic results. We must first use export2graphlan to convert the MetaPhlAn2 results to a format that GraPhlAn can use.

\section{3) Extraction of functional information}

After characterizing the taxonomic profile of each sample, we must determine which genes are expressed and the biological processes involved. To perform functional profiling (Figure 2(c)), we use HUMAnN2 ${ }^{4}$, which is a pipeline to quickly and accurately determine the presence and abundance of functional gene families and pathways from metagenomic or metatranscriptomics data.

For identifying the functions expressed by the community, we filter out the rRNA sequences, due to their high noise levels and the compute time needed for their analysis. The output of SortMeRNA and the identified community profile from MetaPhlAn2 helps HUMAnN2 to focus on the known sequences for the identified organisms. Software tools such as 'Renormalize', 'Unpack Pathway abundance to show gene families', 'Create gene level families file', 'Group abundances' and text manipulation tools such as 'Select lines', 'group columns' and 'sort columns' have been introduced in the ASaiM-MT workflow. This generates normalized abundances of gene families, gene pathways and GO slim terms present in each sample.

4) Combine taxonomic and functional information

The final step (Figure 2(d)) in this analysis is to determine which microorganisms are contributing to the profile of functions indicated by the expressed RNA sequences. HUMAnN2 partially answers this question by including taxa in its gene family and pathway outputs, but it does not include the taxa's abundance, only the functional abundance. We can fill in this missing information with the MetaPhlAn2 results using the Combine MetaPhlAn2 and HUMAnN2 Outputs tools. This produces a table of functional terms and their abundances with the corresponding genus and species abundances for the taxa which contribute to said function via their expressed RNA sequences. The abundances are reported in RPK values (reads per kilobase), calculated as the sum of the scores for all alignments for a gene family. These alignment scores are calculated according to the number of matches of a specific sequence to its reference genome and further normalized to account for multiple reference genome matches.

\section{Results}

To demonstrate the use of the ASaiM-MT workflow, we analyzed a representative metatranscriptomic data set obtained from a microbial community within a thermophilic biogas reactor ${ }^{19}$ which digests municipal food waste and manure (Figure 3). The microbial community was sampled from the bioreactor and transferred to a rich medium containing lignocellulose from Norwegian Spruce and incubated at $65^{\circ} \mathrm{C}$ as an enrichment strategy. Triplicate mRNA samples were taken in a time series from 0 to 43 hours after inoculation. The mRNA was sequenced for paired-end reads ( 2 x $125 \mathrm{bp}$ ) on one lane of an Illumina HiSeq 3000. For the purpose of this study, we took only one time point (8hr). The paired FASTQ files (forward and reverse reads) were then subjected to the ASaiM-MT workflow (Figure 2).

The ASaiM-MT workflow consists of four steps, i) preprocessing of the data, ii) extraction of community profile, iii) extraction of functional information, and iv) combining taxonomic and functional information. The data is preprocessed to make it compatible for MetaPhlAn2 (taxonomy) and HUMAnN2 (Function) annotation of the data.

To extract the taxonomic profile, the MetaPhlAn2 suite was run on the adapter trimmed interlaced files. The Community Profile output contained the information regarding the microbiome community present in the sample along with its relative abundance at different levels, i.e., Kingdom, Phylum, Class, Order, Family, Genus, Species, and Strain (Figure 4). For example - (k Bacteria|p_Firmicutes $\mid \mathrm{c} \_$Clostridia $\mid \mathrm{o} \_$Thermoanaerobacterales $\mid$ f__Thermodesulfobiaceae $\mid g$ __C Coprothermobacter|s__Coprothermobacter_proteolyticus) states that kingdom is bacteria, class is clostridia, belonging to order thermoanaerobacter, family of thermodesulfobiaceae, genus is copthermobacter and species is Comprothermobacter proteolyticus. As it is a cellulose-degrading consortium from anaerobic digestion, Coprothermobacter and Clostridium were expected to be identified for this dataset, demonstrating the accuracy of these tools.

The community profile is further processed using the Format MetaPhlAn2 tool which splits the MetaPhlAn2 output and categorizes them into various taxonomy levels (Kingdom, Phylum, Class, Order, Family, Genus, Species) with corresponding abundance values. Supplementary Figure S1 (Extended data $^{20}$ ) shows genus level relative abundance values associated with genera present in the dataset. For visualization of the taxonomic profile, GraPhlAn and Krona (interactive) were used (Figure $5 \mathrm{a}$ and $5 \mathrm{~b}$ ).

The HUMAnN2 suite of tools were used to extract functional information along with their relative abundance (RPK). 


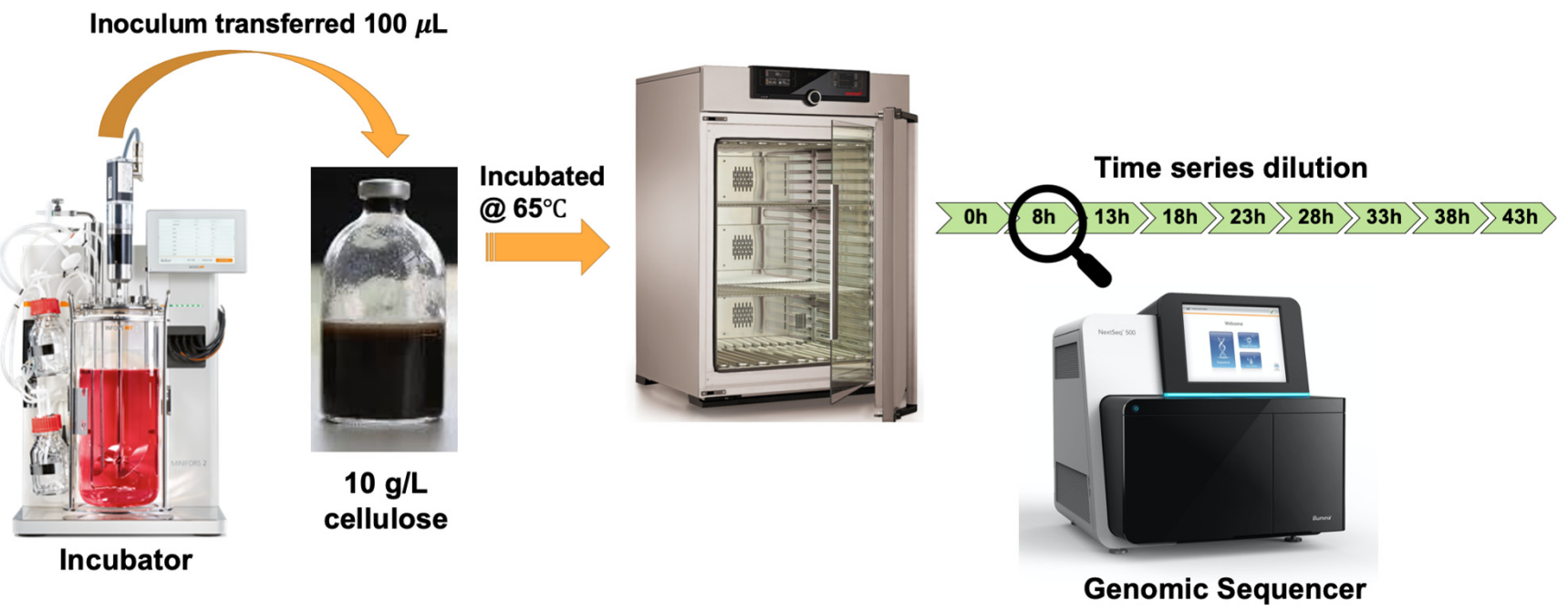

Figure 3. Graphical representation of Biogas reactor Dataset. A $100 \mu l$ inoculum was collected from a lab-scale biogas reactor incubated at $55^{\circ} \mathrm{C}$ and transferred to an anaerobic flask containing $10 \mathrm{~g} / \mathrm{L}$ of cellulose. Triplicate mRNA samples were taken in a time series from 0 to 43 hours after inoculation. Metagenomic and metaproteomic sequencing was performed for all time points. For this tutorial we used one of the triplicates (T1A) in the 8 hours' time point.

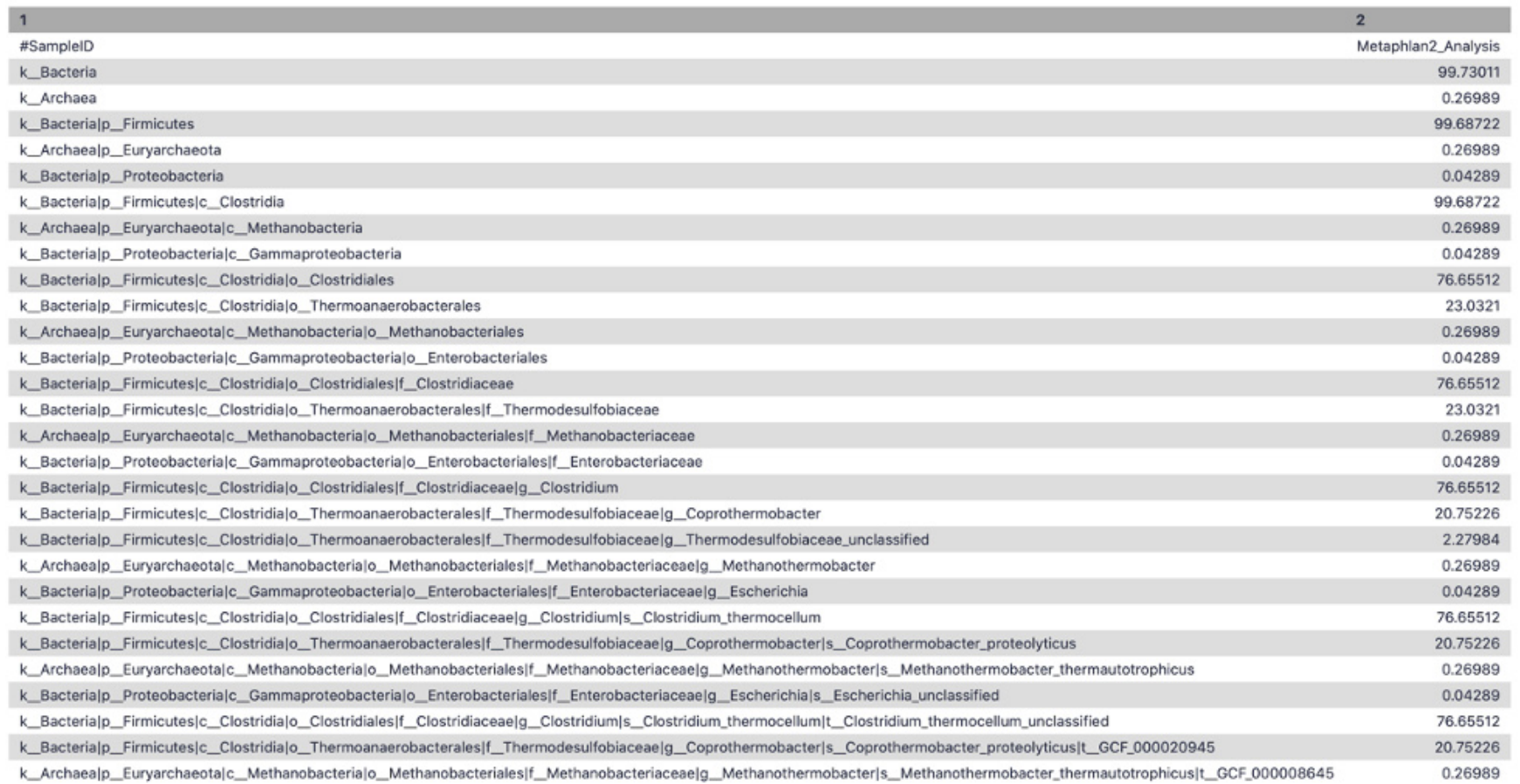

Figure 4. MetaPhIAn2 community profile. A tabular representation of MetaPhIAn2 community profile displaying the different levels of taxonomic classification and its relative abundance at that level.

HUMAnN2 provides 3 different outputs- Gene family and their abundance (Extended data: Supplementary Figure S2a ${ }^{20}$ ), pathways and their abundance (Extended data: Supplementary Figure $\mathrm{S}^{2} \mathrm{~b}^{20}$ ) and pathway and their coverage (Extended data: Supplementary Figure $\mathrm{S} 2 \mathrm{c}^{20}$ ).
In this workflow, the UniRef50 database was used to classify the gene family, but the users also have a choice to use UniRef90. Gene family abundance at the community level is stratified to show the contributions from known and unknown species. The gene family output shows total 

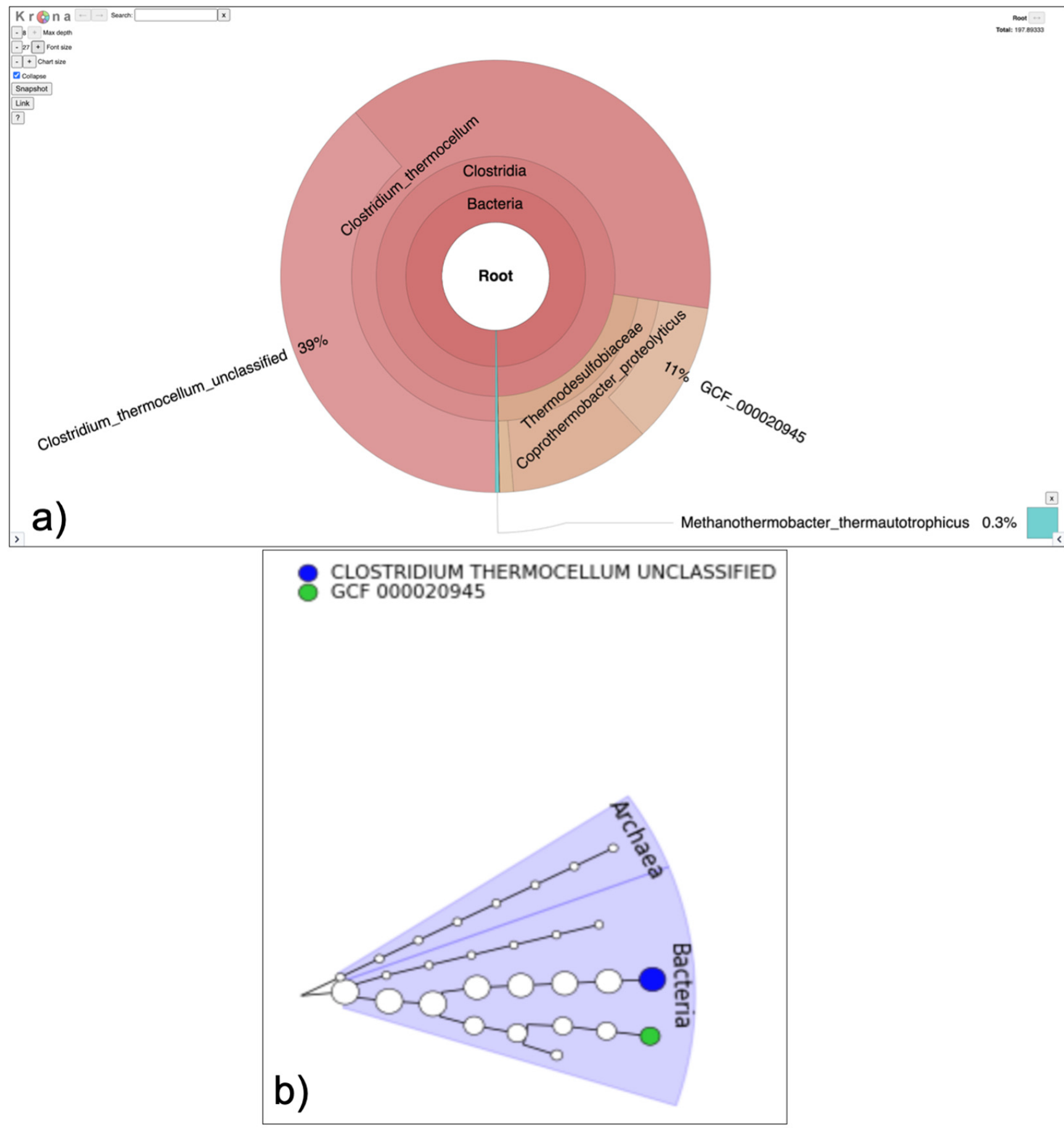

Figure 5. Visualization of Taxonomy output. a) The Krona output provides interactive representation of the community in the sample. In this case Coprothermobacter and Clostridium were the most abundant genera. b) The GraPhIAn outputs circular phylogenetic trees showing that Archaea and bacteria are present in the sample.

abundance value which is the sum total of individual species abundance values (reported as RPK values). Additionally, the tabular output also enlists the contribution of individual species to the gene family abundance (Extended data: Supplementary Figure $\mathrm{S}^{20}$ ). While there are some applications, e.g., strain profiling, where RPK units are superior to depth-normalized units, most of the time we need to renormalize our samples prior to downstream analysis. The gene families can be a long list of IDs and going through the gene families one by one to identify the interesting ones can be cumbersome and error 
prone. To help construct "the bigger picture", we could identify and use categories of genes using the gene families. Gene Ontology (GO) analysis is widely used to reduce complexity and highlight biological processes in genome-wide expression studies. There is a dedicated tool called Group abundances of UniRef50 gene families obtained to gene ontology (GO) slim Terms, which groups and converts UniRef50 gene family abundances generated with HUMAnN2 into GO slim terms (Extended data: Supplementary Figure $\mathrm{S}^{27}$ ) as the name suggests.

The functional and taxonomic annotations from MetaPhlAn2 and HUMAnN2 are further normalized and combined to create a single tabular output.

\section{Use cases}

Here we provide a trimmed version of the biogas reactor dataset to demonstrate the use of the ASaiM-MT workflow in the tutorial available in the GTN.

Link: https://training.galaxyproject.org/training-material/topics/ metagenomics/tutorials/metatranscriptomics/tutorial.html

Trimmed input: https://zenodo.org/record/3362849

Workflow: https://training.galaxyproject.org/training-material/ topics/metagenomics/tutorials/metatranscriptomics/workflows/

\section{Discussion}

The ASaiM-MT workflow is made available in the Galaxy platform via ASaiM, enabling accessibility and flexibility for customization. There are a few tools that can be used alternatively or in addition to the existing tools. The ASaiM-MT workflow (as mentioned in the methods section) was tested to ensure that the workflow works on metatranscriptomics datasets. For details about default parameters used, we recommend visiting the metatranscriptomic tutorial available on the GTN material ${ }^{21}$.

For example, in ASaiM-MT, we map the UniRef 50 values to GO terms, but they can be also mapped to the MetaCyc reactions $^{22}$, KEGG Orthogroups $(\mathrm{KOs})^{23}$, Pfam domains ${ }^{24}$, EC categories ${ }^{25}$ and EggNOG (including COGs) ${ }^{26}$ using the HUMAnN2 regroup tool. A current limitation of the ASaiM-MT workflow is that it can process only one pairedend or single-end data at a time. In order to generate an input for statistical analysis, we have developed an additional postprocessing workflow and Galaxy tool called MT2MQ (https:// github.com/galaxyproteomics/tools-galaxyp/tree/master/tools/ $\mathrm{mt} 2 \mathrm{mq}$ ), which integrates results from multiple outputs from the ASaiM-MT workflow. The MT2MQ workflow combines the gene abundance output from multiple samples or conditions, normalizes the values and makes it compatible with statistical tools such as metaQuantome tool ${ }^{13}$, which can be used for visualizing and interpreting results. Furthermore, we are in the process of developing tools that can help perform multi-omics studies by integrating results from the ASaiM-MT workflow to our existing metaproteomics workflows.

\section{Conclusion}

ASaiM-MT workflow is a robust and extensible Galaxy workflow which is now optimized and tested for metatranscriptomics data. The workflow consists of tested open-source tools in the area of RNA sequence analysis, such as SortMeRNA, MetaPhlAn2 and HUMAnN2. ASaiM-MT in Galaxy offers users a high-level control over their data and provides different analysis options. The GTN offers documentation and resources necessary for new users to gain mastery in using this workflow and associated tools for data analysis for their research projects. ASaiM-MT allows users to not only understand the taxonomy but also the functional composition and pathways expressed by the microbiome present in diverse microbial communities of interest.

\section{Data availability}

\section{Underlying data}

Zenodo: Training Data for "Metatranscriptomics analysis using microbiome RNASeq data", http://doi.org/10.5281/zenodo. $3362849^{27}$.

\section{Extended data}

Zenodo: Supplementary for ASaiM-MT: A validated and optimized ASaiM workflow for metatranscriptomics analysis within Galaxy framework, http://doi.org/10.5281/zenodo.434139120.

This project contains the following extended data:

- Supplementary Figure S1: MetaPhlAn2 Genus-Level Abundance

- Supplementary Figure S2a: HUMAnN2 Gene Family Abundance

- Supplementary Figure S2b: HUMAnN2 Pathway Abundance

- Supplementary Figure S2c: HUMAnN2 Pathway Coverage

- Supplementary Figure S3: Uniref50 Gene Family output with abundance

- Supplementary Figure S4: Conversion of Uniref 50 values to GO terms

Data are available under the terms of the Creative Commons Attribution 4.0 International license (CC-BY 4.0).

\section{Software availability}

Software available from: https://metagenomics.usegalaxy.eu/

Source code available from: https://github.com/ASaiM/framework

Archived source code at time of publication: http://doi.org/ $10.5281 /$ zenodo. $4455627^{28}$.

License: Apache 2 License

Docker: https://quay.io/repository/bebatut/asaim-framework (command: docker pull quay.io/bebatut/asaim-framework). 


\section{Acknowledgements}

We would like to thank European Galaxy team for the help in the support during Galaxy implementation. We would also like to thank Björn A. Grüning (University of Freiburg, Germany) for helping us during the implementation of the workflow in the European Galaxy platform.
1. Mohajeri MH, Brummer RJM, Rastall RA, et al.: The role of the microbiome for human health: from basic science to clinical applications. Eur J Nutr. 2018; 57(Suppl 1): 1-14.

PubMed Abstract | Publisher Full Text | Free Full Text

2. Bolyen E, Rideout JR, Dillon MR, et al.: Author Correction: Reproducible, interactive, scalable and extensible microbiome data science using QIIME 2. (Nature Biotechnology, (2019), 37, 8, (852-857). Nat Biotechnol. Nature Publishing Group; 2019; 37(9): 1091.

PubMed Abstract | Publisher Full Text

3. Schloss PD, Westcott SL, Ryabin T, et al:: Introducing mothur: Open-source platform-independent, community-supported software for describing and comparing microbial communities. Appl Environ Microbiol. 2009; 75(23): 7537-41.

PubMed Abstract | Publisher Full Text | Free Full Text

4. Franzosa EA, McIver LJ, Rahnavard G, et al.: Species-level functional profiling of metagenomes and metatranscriptomes. Nat Methods. 2018; 15(11): 962-968.

PubMed Abstract | Publisher Full Text | Free Full Text

5. Turnbaugh PJ, Gordon JI: An Invitation to the marriage of metagenomics and metabolomics. Cell. 2008; 134(5): 708-13. PubMed Abstract | Publisher Full Text

6. Bashiardes S, Zilberman-Schapira G, Elinav E: Use of metatranscriptomics in microbiome research. Bioinform Biol Insights. 2016; 10: 19-25.

PubMed Abstract | Publisher Full Text | Free Full Text

7. Wilmes $\mathrm{P}$, Bond $\mathrm{PL}$ : Metaproteomics: Studying functional gene expression in microbial ecosystems. Trends Microbiol. Trends Microbiol; 2006; 14(2): 92-7. PubMed Abstract | Publisher Full Text

8. Shakya M, Lo CC, Chain PSG: Advances and challenges in metatranscriptomic analysis. Front Genet. Frontiers Media S.A.; 2019; 10: 904. PubMed Abstract | Publisher Full Text | Free Full Text

9. ASaiM: an environment to analyze intestinal microbiota data. ASaiM 0.1 documentation. [cited 2020 Dec 17]. Reference Source

10. Giardine B, Riemer C, Hardison RC, et al.: Galaxy: A platform for interactive large-scale genome analysis. Genome Res. 2005; 15(10): 1451-5. PubMed Abstract | Publisher Full Text | Free Full Text

11. Batut B, Hiltemann S, Bagnacani A, et al.: Community-Driven Data Analysis Training for Biology. Cell Syst. 2018; 6(6): 752-758.e1. PubMed Abstract | Publisher Full Text | Free Full Text

12. Afgan E, Baker D, Batut B, et al.: The Galaxy platform for accessible, reproducible and collaborative biomedical analyses: 2018 update. Nucleic Acids Res. 2018: 46(W1): W537-W544. PubMed Abstract | Publisher Full Text | Free Full Text

13. Easterly CW, Sajulga R, Mehta S, et al.: MetaQuantome: An integrated, quantitative metaproteomics approach reveals connections between taxonomy and protein function in complex microbiomes. Mol Cell Proteomics. 2019; 18(8 suppl 1): S82-S91.

PubMed Abstract | Publisher Full Text | Free Full Text

14. Martin M: Cutadapt removes adapter sequences from high-throughput sequencing reads. EMBnet J. 2011; 17(1): 10.

Publisher Full Text

15. Kopylova E, Noé L, Touzet H: SortMeRNA: Fast and accurate filtering of ribosomal RNAs in metatranscriptomic data. Bioinformatics. 2012; 28(24): 3211-7.

PubMed Abstract | Publisher Full Text

16. Truong DT, Franzosa EA, Tickle TL, et al.: MetaPhIAn2 for enhanced metagenomic taxonomic profiling. Nat Methods. Nature Publishing Group; 2015; 12(10): 902-3.

PubMed Abstract | Publisher Full Text

17. Ondov $\mathrm{BD}$, Bergman $\mathrm{NH}$, Phillippy AM: Interactive metagenomic visualization in a Web browser. BMC Bioinformatics. 2011: 12.385. PubMed Abstract | Publisher Full Text | Free Full Text

18. Asnicar F, Weingart G, Tickle TL, et al.: Compact graphical representation of phylogenetic data and metadata with GraPhIAn. PeerJ. 2015; 3: e1029. PubMed Abstract | Publisher Full Text | Free Full Text

19. Kunath $B J$, Delogu $F$, Naas $A E$, et al.: From proteins to polysaccharides: lifestyle and genetic evolution of Coprothermobacter proteolyticus. ISME J. 2019; 13(3): 603-617.

PubMed Abstract | Publisher Full Text | Free Full Text

20. Mehta S: Supplementary for ASaiM-MT: A validated and optimized ASaiM workflow for metatranscriptomics analysis within Galaxy framework. 2020.

http://www.doi.org/10.5281/zenodo.4341391

21. Metatranscriptomics analysis using microbiome RNA-seq data. [cited 2020 Nov 25]. Reference Source

22. Caspi $R$, Billington $R$, Fulcher $C A$, et al: The MetaCyc database of metabolic pathways and enzymes. Nucleic Acids Res. 2018; 46(D1): D633-D639. PubMed Abstract | Publisher Full Text | Free Full Text

23. Kanehisa $\mathrm{M}$, Sato $\mathrm{Y}$, Kawashima M, et al.: KEGG as a reference resource for gene and protein annotation. Nucleic Acids Res. 2016; 44(D1): D457-62. PubMed Abstract | Publisher Full Text | Free Full Text

24. Finn RD, Bateman A, Clements J, et al.: Pfam: The protein families database. Nucleic Acids Res. Nucleic Acids Res; 2014; 42(Database issue): D222-30. PubMed Abstract | Publisher Full Text | Free Full Text

25. Dönertaş HM, Cuesta SM, Rahman SA, et al.: Characterising complex enzyme reaction data. PLoS One. 2016; 11(2): e0147952. PubMed Abstract | Publisher Full Text | Free Full Text

26. Huerta-Cepas J, Szklarczyk D, Heller D, et al.: EggNOG 5.0: A hierarchical, functionally and phylogenetically annotated orthology resource based on 5090 organisms and 2502 viruses. Nucleic Acids Res. 2019; 47(D1): D309-D314. PubMed Abstract | Publisher Full Text | Free Full Text

27. Batut $B$, Mehta S, Kumar P: Training Data for "Metatranscriptomics analysis using microbiome RNASeq data" [Data set]. Zenodo. 2019 http://www.doi.org/10.5281/zenodo.3362849

28. Batut B, Grvl K, Hiltemann S, et al.: ASaiM/framework: ASaiM-MT release (Version ASaiM-MT). Zenodo. 2021. http://www.doi.org/10.5281/zenodo.4455627 


\section{Open Peer Review}

\section{Current Peer Review Status: ?}

Version 1

Reviewer Report 18 February 2021

https://doi.org/10.5256/f1000research.31653.r79463

(C) 2021 Simopoulos C. This is an open access peer review report distributed under the terms of the Creative Commons Attribution License, which permits unrestricted use, distribution, and reproduction in any medium, provided the original work is properly cited.

\section{Caitlin Simopoulos}

Department of Biochemistry, Microbiology and Immunology, University of Ottawa, Ottawa, ON, Canada

The authors describe an optimized workflow for metatranscriptomic microbial community data named ASaiM-MT. ASaiM-MT is an extension of ASaiM, a workflow mostly focused on metagenomic data available via Galaxy. Using helpful diagrams, the authors describe how ASaiMMT differs from the original ASaiM workflow, and detail which tools from the Galaxy platform are used. Finally, the authors use data from a biogas reactor study the pipeline and demonstrate results that can be expected using ASaiM-MT. I particularly enjoyed the fact that the article is accompanied by written training material for the workflow. I believe that tutorials and userfriendly tools expand the audience of traditionally "bioinformatician-only" tools. However, I have a few suggestions for the authors to strengthen their presentation of the metatranscriptomic workflow before publication.

\section{Major comments:}

1. In your discussion, you highlighted a major limitation of ASaiM-MT: the fact that it can only handle a single sample and does not complete comparative analysis. However, you also mention that you have developed post-processing tools for this reason. It might be good to highlight that ASaiM-MT is essential (in particular for data pre-processing and identification of functional and taxonomic information) to be completed before statistical analysis. In addition, is it possible to process data in ASaiM-MT in batches? This might also lessen the perceived limitation of the tool.

2. Sometimes it is not completely clear why ASaiM-MT is needed if ASaiM was developed for both meta-genomics and -transcriptomics. It is important to be specific and highlight the rationale for developing the ASaiM-MT workflow. An example: "For performing this action, the original ASaiM Shotgun workflow used the FASTQ-joiner to join the reads. However, in the ASaiM-MT version, we use the FASTQ interlacer. FASTQ interlacer joins the forward (/1) and the reverse reads (/2) using the sequence identifiers; sequences without designation will be named as single reads. The reason ASaiM-MT uses FASTQ-interlacer rather than FASTQ-joiner is because the 
joiner tool combines the forward and reverse read sequence together while the interlacer puts the forward and reverse read sequences in the same file while retaining the entity of each read along with an additional file with unpaired sequences." Was this change made because FASTQ interlacer is more appropriate for RNA sequencing, or was it a change to improve on the original ASaiM workflow?

3. The abstract and introduction seem to emphasize the importance of studying the human microbiome and its connections to health and well-being, however, this manuscript uses a microbial community obtained from a biogas reactor. This is a very interesting community that warrants further research, but the introduction does not match the sample of interest. I suggest updating the introduction to focus less on the human microbiome and emphasize the importance of studying microbial communities in general. Alternatively, if you'd like to focus on human microbiomes, the example data used should reflect the human focus.

4. In your discussion, you say: "There are a few tools that can be used alternatively or in addition to the existing tools." Does this mean that there are other tools like ASaiM-MT that can be used? Your introduction mainly listed tools that complete specific tasks and not an end to end workflow. If there are other workflow tools, a comparison should be highlighted in the discussion or introduction. What makes ASaiM-MT unique compared to other tools/workflows? What are its strengths? Is it more user-friendly than other tools? Expanding on this will help strengthen your conclusions about the tool itself.

\section{Minor comments:}

1. There are sections that can read like a "grocery list" of software names (eg. second paragraph of the introduction). I understand why you are listing them, but it could be useful to emphasize that each of these tools performs a single task and need to be put together into a workflow for a complete experiment. These tools are also often missing citations.

2. It can be difficult to understand which words are actually software names. Is it possible to type them in a monospace font? Or to bold the names?

3. At times the "Methods" section can get confusing, particularly when MT is being compared to the original ASaiM. My suggestion is to include numbers or roman numerals in the "in between" steps as described in Figure 2. That way you can also reference them in the text. For example, in "a) Preprocessing" there would be i. Input files, ii. Quality Control, iii. Adapter Trimming... etc.. It might also be useful to include these headers (and numbers/letters) in your Methods section to let readers follow along.

4. "PMID 30298254" is used instead of a citation

Is the rationale for developing the new method (or application) clearly explained? Partly

Is the description of the method technically sound? Partly

Are sufficient details provided to allow replication of the method development and its use 
by others?

Yes

If any results are presented, are all the source data underlying the results available to ensure full reproducibility?

Yes

Are the conclusions about the method and its performance adequately supported by the findings presented in the article?

Partly

Competing Interests: No competing interests were disclosed.

Reviewer Expertise: Computational biology, bioinformatics, RNA-sequencing data pipeline development and analysis, microbiomes

I confirm that I have read this submission and believe that I have an appropriate level of expertise to confirm that it is of an acceptable scientific standard, however I have significant reservations, as outlined above.

Author Response 25 Mar 2021

Subina Mehta

We greatly appreciate the reviewer's comments and suggestions. We have incorporated the required changes in the updated version of the manuscript.

\section{Major comments:}

1. In your discussion, you highlighted a major limitation of ASaiM-MT: the fact that it can only handle a single sample and does not complete comparative analysis. However, you also mention that you have developed post-processing tools for this reason. It might be good to highlight that ASaiM-MT is essential (in particular for data pre-processing and identification of functional and taxonomic information) to be completed before statistical analysis. In addition, is it possible to process data in ASaiM-MT in batches? This might also lessen the perceived limitation of the tool.

A: We would like to thank the reviewer for the comment. We have edited the abstract and mentioned that ASaiM-MT is essential for preprocessing and identification of taxonomy and functional information before performing comparative analysis. Also, Galaxy has a function of providing datasets as a collection rather than a single input which can be used to handle multiple datasets. In the Galaxy platform, we have other software tools such as MT2MQ and metaQuantome, which can perform comparative statistical analysis.

2. Sometimes it is not completely clear why ASaiM-MT is needed if ASaiM was developed for both meta-genomics and -transcriptomics. It is important to be specific and highlight the rationale for developing the ASaiM-MT workflow. An example: "For 
performing this action, the original ASaiM Shotgun workflow used the FASTQ-joiner to join the reads. However, in the ASaiM-MT version, we use the FASTQ interlacer. FASTQ interlacer joins the forward (/1) and the reverse reads (/2) using the sequence identifiers; sequences without designation will be named as single reads. The reason ASaiM-MT uses FASTQ-interlacer rather than FASTQ-joiner is because the joiner tool combines the forward and reverse read sequence together while the interlacer puts the forward and reverse read sequences in the same file while retaining the entity of each read along with an additional file with unpaired sequences." Was this change made because FASTQ interlacer is more appropriate for RNA sequencing, or was it a change to improve on the original ASaiM workflow?

A: Thank you for this comment. We replaced the FASTQ-joiner tool with the FASTQ interlacer to improve on the original workflow. The FASTQ interlacer tool maintains the integrity of reads by maintaining the forward and the reverse sequence identifiers, as compared to joining them into a single read file, as is done by FASTQ-joiner tool.

3. The abstract and introduction seem to emphasize the importance of studying the human microbiome and its connections to health and well-being, however, this manuscript uses a microbial community obtained from a biogas reactor. This is a very interesting community that warrants further research, but the introduction does not match the sample of interest. I suggest updating the introduction to focus less on the human microbiome and emphasize the importance of studying microbial communities in general. Alternatively, if you'd like to focus on human microbiomes, the example data used should reflect the human focus.

A: We thank the reviewer for the comment and have edited the abstract and introduction to highlight the importance of microbiome research in ecology as well as clinical research.

4. In your discussion, you say: "There are a few tools that can be used alternatively or in addition to the existing tools." Does this mean that there are other tools like ASaiMMT that can be used? Your introduction mainly listed tools that complete specific tasks and not an end to end workflow. If there are other workflow tools, a comparison should be highlighted in the discussion or introduction. What makes ASaiM-MT unique compared to other tools/workflows? What are its strengths? Is it more user-friendly than other tools? Expanding on this will help strengthen your conclusions about the tool itself.

A: We thank the reviewer for the comment. The tools mentioned in the discussion offer an alternative option to the tools that we have used in the AsaiM-MT workflow, especially if the users have a preference. However, although we consider these tools to be appropriate to metatranscriptomics research, we have not tested these tools.

ASaiM-MT workflow, due to its availability in Galaxy, offers an user-friendly option to the existing command line tools. The ASaiM-MT workflow has been tested with different datasets to ensure its compatibility. There is a systematic documentation available for the usage of the workflow in the Galaxy Training Network (GTN). Users can also ask questions to developers and users via the Gitter channel, if needed. 


\section{Minor comments:}

1. There are sections that can read like a "grocery list" of software names (eg. second paragraph of the introduction). I understand why you are listing them, but it could be useful to emphasize that each of these tools performs a single task and need to be put together into a workflow for a complete experiment. These tools are also often missing citations.

A: Thanks to the reviewer for the comment. The tools listed are alternatives to the existing tools in the workflow and have not been incorporated as a workflow. We have added citations to these tools.

2. It can be difficult to understand which words are actually software names. Is it possible to type them in a monospace font? Or to bold the names?

A: Thanks to the reviewer for this comment. I have formatted the tool names by making them italic.

3. At times the "Methods" section can get confusing, particularly when MT is being compared to the original ASaiM. My suggestion is to include numbers or roman numerals in the "in between" steps as described in Figure 2. That way you can also reference them in the text. For example, in "a) Preprocessing" there would be i. Input files, ii. Quality Control, iii. Adapter Trimming... etc.. It might also be useful to include these headers (and numbers/letters) in your Methods section to let readers follow along.

A: Thank you for the comment. I have reformatted the method section according to Figure 2.

4. "PMID 30298254" is used instead of a citation

A: Thank you reviewer for pointing this out. I have made the required change.

Competing Interests: No competing interests were disclosed. 
The benefits of publishing with F1000Research:

- Your article is published within days, with no editorial bias

- You can publish traditional articles, null/negative results, case reports, data notes and more

- The peer review process is transparent and collaborative

- Your article is indexed in PubMed after passing peer review

- Dedicated customer support at every stage

For pre-submission enquiries, contact research@f1000.com 